Психология. Журнал Высшей школы экономики,

2021. T. 18. № 3. C. 525-543. DOI: 10.17323/1813-8918-2021-3-525-543

\title{
СИТУАЦИОННЫЙ ПОДХОД: ТИПЫ СИТУАЦИЙ И ПСИХОЛОГИЧЕСКИЕ ОСОБЕННОСТИ
}

\section{Г.Н. СОЛНЦЕВА}

${ }^{a}$ Московский государственный университет имени М.В. Ломоносова, 119991, Россия, Москва, Ленинские горы, 1

\section{Резюме}

В широком спектре психологических подходов перспективным представляется ситуационный подход, получивший теоретическое обоснование в отечественных исследованиях. Центральным является понятие ситуации как опосредующего элемента между условиями среды и индивидуально-личностными особенностями субъекта в контексте проблем двойной детерминации деятельности. Определения ситуации преимущественно ограничиваются представлением о субъективном образе внешних условий, адекватность которого интерпретируется как проявление средовой, а вариативность - проявление личностной детерминации деятельности. Пристрастность образа внешних условий является одним из проявлений личностной детерминации когнитивной составляющей, но не снимает проблемы детерминации деятельности. Цель работы - ограничение понятия «ситуация» субъективной сферой и его интерпретация как результата интеграции детерминант. Уточнены представления об условиях среды, аргументирована необходимость оценок интерпретируемого образа условий, собственных ресурсов и их соотношения, а также «пространства» оценок как механизма интеграции детерминант. Ситуация определяется как рефлексивная оценочная «модель» отношений интерпретируемого образа объективных условий и внутреннего состояния в пределах актуальной мотивации, наличного опыта и личностных предпочтений. Функции ситуации - организация деятельности (актуалгенез) и ее регуляция на стадии исполнения, обеспечивающие ее стабильность и целесообразность. Представлена систематизация ситуаций на основе типологии условий и вариантов их субъективных оценок, наличных ресурсов субъекта и их оценок. Особое внимание уделено ситуациям неопределенности, принятия решения и риска, определены их особенности, психологические механизмы и личностные детерминанты. Ситуационный подход расширяет возможности объяснения вариативности деятельности в тождественных условиях, ее устойчивость в меняющихся условиях, а также объяснение причин ошибок, обусловленных своеобразием оценок рефлексивной модели реальности.

Ключевые слова: среда, условия, ситуация, чрезвычайные условия, сложность, экстремальность, опасность, напряженность, неопределенность, проблемность, принятие решения, риск, рефлексия.

\section{Понятие ситуации и ситуационный подход в системе психологического знания}

Понятие ситуации формировалось в русле проблемы двойной детерминации деятельности внешними условиями и личностными особенностями, 
решение которой связывается с идеей взаимодействия человека со средой, в ходе и благодаря которому достигается соответствие внешних условий и внутренних переменных, обеспечивающих целесообразность действий. Вопрос не в том, какие переменные в большей мере определяют особенности деятельности и поведения - внешние средовые или внутренние «личностные». Проблема в том, каким образом осуществляется взаимодействие столь различных реальностей, каков механизм их «интеграции» и в какой форме результат репрезентируется субъекту, обеспечивая стабильность деятельности до момента получения результата в меняющихся условиях и индивидуальную вариативность в схожих условиях. Ситуационный подход в своем первоначальном виде базировался на представлении о решающей роли «ситуации как совокупности внешних обстоятельств» в противовес «персонологическому», в котором индивидуально-личностные особенности рассматривались как основная детерминанта деятельности, не снимая проблемы двойной детерминации. Ситуационный подход может рассматриваться как вариант интеракционистского с сохранением центральной идеи взаимодействия человека и среды как детерминанты деятельности, дополненной идеей опосредованного характера взаимодействия. В качестве опосредующего элемента рассматривается ситуация, которая должна обладать свойствами обеих сторон взаимодействия - быть конгруэнтной как актуальным условиям среды, так и состоянию человека. Функция ситуации - интеграция актуальных переменных, что снимает обсуждение вопроса о приоритете средовой или личностной детерминанты.

В таком понимании первостепенной задачей ситуационного подхода является уточнение понятия «ситуация». Имеющиеся определения фиксируют значимые, но разрозненные признаки ситуации, не отвечают логическим нормам - однозначности отнесения к более широкой категории, фиксации отличий и связей схожих явлений внутри категории, снижая определенность представлений, смысл подхода и объяснительный потенциал.

Определения и анализ ситуации достаточно широко представлены в литературе, в том числе в работах теоретического и обзорного характера (Гришина, 2008; Осухова, 2012; Попова, 2011; Рягузова, 2006; Трифонова, 2004). Понятие ситуации часто ограничивается совокупностью элементов среды, трактуется как отдельное «событие» во внешней среде или совокупность условий (обстоятельств), а личностная обусловленность деятельности рассматривается как константа. Отнесение ситуации к элементам среды не только обесценивает идею опосредования, но и приводит к определениям, в которых ситуация «оказывается» «единством личности и ситуации», результатом «взаимной обусловленности ситуации и человека» или крайне неопределенным «нечто», с чем взаимодействует личность. Определение ситуации как «системы» тех же субъективных и объективных элементов, но в деятельности (Ломов, 1999), задало в свое время общие ориентиры ее содержательного анализа. В ряде работ представление о ситуации приобрело признаки системности за счет включения категорий активности субъекта. Ситуация описывается как концептуализация взаимодействий человека со средой (Филиппов, Ковалев, 
1986) или когнитивный конструкт личности, отражающий часть объективной реальности (Воронин, Князев, 1989), как внутренние процессы, сопряженные с внешним пространством, интегрирующие всю совокупность детерминант поведения (Анцупов, Шипилов, 2010). Эти определения фиксируют принципиальные характеристики ситуации - принадлежность субъективно-личностной сфере и ее интегрирующую функцию, требующие, однако, конкретизации в системе деятельности и психологических категорий ее описания.

Понятие ситуации, ограниченное сферой психических явлений, предполагает не только трансформацию и интерпретацию «предметности» актуальных условий среды в субъективный образ, личностно пристрастный когнитивный конструкт. Решающими для субъективного «определения» ситуации являются преодоление «непосредственности» восприятия условий и формирование отношения к ним - оценки в актуальный момент деятельности. Следует подчеркнуть, что личностная пристрастность оценки не ограничивается влиянием индивидуально-личностных особенностей, с необходимостью приобретает форму оценки собственного состояния (ресурсов), что обеспечивает сопоставление и интеграцию детерминирующих переменных. Ситуация для субъекта предстает в форме оценки отношений интерпретируемых внешних условий и внутренних ресурсов, что предполагает более высокий - рефлексивный уровень регуляции деятельности, а для психологического анализа определяется как рефлексивная оценочная «модель» отношений объективных условий и внутреннего состояния.

Анализ закономерностей формирования ситуации как рефлексивной модели требует уточнения представлений о среде и ее составляющих. Понятие среды ограничивается внешним окружением, не распространяется на характеристики внутренних процессов человека, которые обозначаются как состояние. Внешние детерминанты деятельности обычно представлены общими понятиями среды, описываются как обстоятельства, события, создающие определенные условия, в которых вынужден действовать субъект. Среда всегда рассматривается как противопоставление человеку, приобретает определенность только по отношению к субъекту, все проявления активности человека являются средовыми, но не все параметры среды влияют на характеристики деятельности. Среда понимается как совокупность взаимосвязанных переменных объективной реальности, имеющих разные пространственно-временные границы. Устоявшимся является представление о составляющих физической среды - материальных объектах, их закономерных взаимосвязях, проявляющихся в изменчивости характеристик объектов. Социальная среда описывается в терминах относительно устойчивого окружения, ограниченного пространственной и социальной близостью, а также социальных условий как характеристик связей актуального окружения и их изменений в отдельных эпизодах (событиях) (Магнуссон, 1983). Для физической и социальной среды представление об условиях целесообразно ограничить изменчивыми параметрами, характеризующими актуальность и требующими учета в организации деятельности. Информационная среда также противопоставляется человеку, но представления о ней не столь однозначны: не имеют пространственно-временной 
определенности, описывается как сеть или «киберпространство» коммуникаций, в котором трудно выделить «объективные» устойчивые или вариативные характеристики.

Только в рамках бихевиоризма условия могут рассматриваться как детерминанта, в других концепциях условия как характеристики среды, противопоставленные человеку, сами по себе не являются детерминантами, должны приобрести качество «субъектности» - отражены субъектом, сохранив тождественность условиям. Но и образ реальности не может непосредственно влиять на схемы поведения, пока не будет включен в систему связей компонентов деятельности, посредством которой обеспечивается целесообразная реорганизация образа, опосредование связей условий, индивидуально-личностных особенностей, актуальной мотивации и опыта. Не столько «субъективный образ» актуального фрагмента окружающей действительности (Трифонова, 2004), сколько «пространство отношений» условий среды и субъективно-личностных характеристик действующего субъекта (Осухова, 2012 ) определяется как ситуация, опосредуя связи и определяя направления «реконструкции и репрезентации» субъектом внешних условий. Еще К. Левин подчеркивал, что поведение определяется целостностью «поля» взаимодействия, в котором условия среды представлены в виде «сконструированной» реальности, релевантной субъективным устремлениям человека. Реконструированная реальность - не «чисто внешнее объективное обстояние, взятое безотносительно к состоянию субъекта, но и не состояние, взятое вне отношения к объектам», «единство обстояния и состояния» и есть ситуация (Василюк, 2003, с. 157).

Поле взаимодействия может быть представлено «пространством непространственных явлений» - сознанием или рефлексией, опосредующим звеном между субъектом и внешней реальностью - моделью, схемой отношений (Лефевр, 2003), что «приводит к четкому разграничению знания и отражаемой реальности» (Швырев, 1985). Только в этом пространстве возможно одновременное сосуществование моделей объективной реальности, включая модель «другого» и полноту репрезентации внутреннего мира во всем многообразии его проявлений (Карпов, 2004), возможно манипулировать образами-моделями - «остановить мгновение», обратиться к прошлому и конструировать будущее. Рефлексия как «посредник» - единственный способ объяснения единства объективного и субъективного, актуального и потенциального, прошлого (опыт) и будущего (результат), рационального и чувственного, индивидуального и социального в деятельности.

Рефлексивное пространство - система иерархически организованных «зеркал» (в метафоре Лефевра) в соответствии с актуальной целью и наличным опытом, ограниченная устойчивыми личностными качествами. Содержание «экрана» - образы актуального фрагмента окружающей действительности и Я субъекта, их взаимное расположение отражает их взаимодействие. На каждом уровне рефлексии актуализируются и получают конкретность образы-модели внешних условий и образы Я - варианты наличных, возможных, желаемых и требуемых состояний личности. Те и другие 
модели приобретают схожие качества - организуются на основе оценки значимости составляющих, устойчивость. Значимость характеристик условий и состояния определяет интерпретацию «образа», «вектор» активности, объем и границы ситуации. Значимые характеристики условий приобретают пространственную независимость, включаются в более широкий временной интервал - соотносятся с прошлыми ситуациями и включаются в ожидаемые (Трифонова, 2004), мотивационные особенности ограничивают включаемые в модель характеристики ресурсов. Модель внешних меняющихся условий, сохраняя адекватность объективному окружению, приобретает устойчивость, как и модель актуального состояния Я в силу устойчивости не столько личностных качеств, сколько актуальной мотивации и наличного опыта. В рефлексивном пространстве эти модели соотносятся - оцениваются, реорганизуются, достигается их субъективное соответствие, которое является критерием и моментом перехода к реализации действия, приобретающего целесообразность и смысл.

Таким образом, ситуация понимается как реконструированная в рефлексивном пространстве система отношений оценок актуальных условий и личностных ресурсов, рефлексивная модель отношений оценок актуальных условий и состояния Я, основанная на оценках значимости и придающая целесообразность и смысл действиям.

\section{Типология и психологические закономерности ситуаций}

Типы ситуаций традиционно связываются с внешними условиями. Диапазон средовых условий достаточно широк, можно ограничиться двумя полюсами их характеристик. Интегральной характеристикой условий является их определение как нормальных, с одной стороны, и особых, с другой. Нормальными признаются условия, параметры которых соответствуют ограниченному диапазону жизнеобеспечения и деятельности (Лебедев, 1989), они отличаются относительным постоянством, высокой вероятностью повторения, описываются как «стандартные», «детерминированные», «хорошо структурированные», «сильные» при узком диапазоне интерпретации. Деятельность в нормальных условиях отличается оптимальным уровнем общей активности и контроля, определенностью и стабильностью регуляции психических процессов на основе сложившихся когнитивных и исполнительных схем действий, обеспечивающих готовность и устойчивость деятельности, снижением факторов мотивации и функционального состояния.

Изменения среды - локальные или глобальные, кратковременные или постоянные, широкого или узкого диапазона, стремительные или постепенные - могут превратить нормальные условия в особые, которым не соответствуют сложившиеся стереотипы действий (Лысаков и др., 2013). Значительные, отличающиеся внезапностью изменения условий на ограниченной территории, источники которых известны, предсказуемы последствия для среды и населения, обозначаются как чрезвычайные. Эти условия возникают в результате действия природных или социальных явлений, техногенных 
происшествий, применения средств поражения, влекущего за собой человеческие жертвы, ущерб здоровью людей или окружающей среде, материальные потери (Шойгу, 2019). Опасные условия - наличие угроз жизни и здоровью человека - характеризуются несоответствием параметров среды узкому диапазону возможностей жизнеобеспечения. Условия опасности включают природные и антропогенные (техногенные, организационные) факторы, человеческий фактор проявляется в ошибочных и несанкционированных действиях, отклонениях характеристик действий от норм и требований. Взаимодействие человека со средой всегда сопряжено с опасностью, поскольку не существует отдельно «хороших» и «плохих» факторов среды, а действие позитивных не исключает генерирования опасных и вредных факторов, что отражено в аксиоме потенциальной опасности как универсального свойства взаимодействия и касается любого вида деятельности.

Возникновение чрезвычайных и опасных условий обусловлено схожими причинами, любые чрезвычайные условия опасны, но опасные не всегда являются чрезвычайными. Чрезвычайные условия преимущественно обусловлены природными и техногенными причинами, всегда актуальны, имеют широкомасштабные негативные последствий для населения определенной территории. Опасные условия могут возникать в результате действий человека по обслуживанию и эксплуатации технических средств и систем, могут носить потенциальный характер, касаются отдельного человека или групп, могут иметь четкие или неопределенные временны́е границы. Чрезвычайные и опасные условия являются экстремальными по отношению к субъекту, характеризуют пространственно-временные параметры среды «события на ограниченной территории», в которых человек оказывается на «персональном пороге» своих адаптивных возможностей (Лебедев, 1989).

К объективным условиям часто относят сложность, определяемую числом объектов, их размерностью, неоднородностью, характером взаимосвязей, динамикой изменений. Трудно согласиться с тем, что сложность является атрибутивной характеристикой среды, существуют простые и сложные характеристики. Сложность зависит от характера познания, является скорее оценкой интерпретируемых условий, чем их характеристикой: чем больше параметров оценены как значимые, тем больше вариантов их интерпретации и задача представляется сложной, затрудняющей оценку значимости, как и при ограниченности опыта. Оценка условий зависит от когнитивных особенностей субъекта, получивших в психологии определение когнитивной сложности. Высокая когнитивная сложность предполагает владение и предпочтения в использовании множества конструктов, имеющих многообразные связи, субъект способен менять основания классификации и воспринимать условия (и себя) по-разному, манипулируя и трансформируя значимые признаки. В зависимости от личностной когнитивной сложности условия могут интерпретироваться с использованием как сложных, так и простых приемов, а простые - неоправданно усложненными способами.

Типы условий не совпадают с типами ситуаций в силу неоднозначности их оценок даже при тождественности интерпретации. Ситуации могут оцениваться 
как обычные или необычные (особые) в зависимости от опыта, восприятие условий может оказаться иллюзорным, но и адекватное восприятие может интерпретироваться по-разному в зависимости от актуальной мотивации. Экстремальные, чрезвычайные, опасные условия могут адекватно восприниматься, но оцениваться как обычные, а обычные привычные условия могут оцениваться как трудные, задачи - как сложные при изменении состояния субъекта. Аналогичные закономерности характеризуют и оценки субъектом своих возможностей - ресурсов действий в актуальных условиях, состояния функциональных систем и опыта. Ресурсы могут в разной степени обеспечить достижение цели: от полного соответствия до рассогласования, а субъективная оценка ресурсов может быть адекватной (субъект осознает и оценивает ограниченность возможностей) или неадекватной. Все ситуации ограниченности ресурсов, адекватной или заниженной оценки, связанные с трудностями использования сложившегося обычного опыта, приобретают характер экстремальных.

Обычные условия, определяемые опытом их интерпретации, адекватной оценкой возможностей действий, не исключают экстремальных ситуаций; для опытного профессионала ситуация может стать экстремальной при изменении функционального состояния, мотивации и значимости отдельных объектов, ограничивающих реализацию имеющегося потенциала. Особые условия могут не сопровождаться экстремальностью за счет сложившихся и отработанных способов действий. Если деятельность осуществляется постоянно в опасных или чрезвычайных условиях, отношение к ним являе тся привычным, ресурсы адекватно оцениваются, ситуация не является экстремальной.

Типы ситуаций могут быть выделены по характеристикам: тип условий нормальные/особые, оценка условий - обычные/экстремальные, ресурсы наличие/ограниченность, оценка ресурсов - адекватная/неадекватная. Крайние типы ситуаций можно описать следующим образом. Первый тип нормальные обычные условия, субъект располагает ресурсами и адекватно их оценивает. Другая крайность - особые условия, неадекватная их оценка, ограниченность ресурсов действий и неадекватная их оценка.

Опасные ситуации возникают преимущественно в опасных условиях при осознании степени угроз и их последствий, адекватной оценке ограниченности ресурсов или заниженной их оценке, ограниченности или отсутствии опыта интерпретации условий и действий в них. Однако угрозы могут не осознаваться, субъект может не знать о них или знать, но не придавать им значения, оценка своих возможностей может оказаться адекватной или завышенной; в этих случаях опасные условия не приобретают характера опасной ситуации. При отсутствии опасных условий и явных угроз субъект может продуцировать «мнимые» угрозы, потенциальные угрозы интерпретировать как реальные, придавать им высокую значимость; ситуация для него представляется опасной вне зависимости от оценки собственных ресурсов. Неадекватная завышенная оценка угроз с приданием им высокой значимости приводит к изменению состояния субъекта, нарушению деятельности вплоть до отказа, ситуация оценивается как предельно опасная. Заниженная оценка 
(вплоть до полного игнорирования) способствует «определению» ситуации как обычной и реализации стандартных для обычных условий приемов действий, лишь вероятностно обеспечивающих успешность. Причины неадекватного восприятия опасности сводятся к ограниченности знаний (опыта), недостоверности информации, ошибкам восприятия и заблуждениям при прогнозировании динамики и последствий реальной обстановки. Принципиальное значение имеют личностные качества субъекта, его самооценка, особенности целевых установок и социальных ориентаций, подверженность влиянию других лиц и рефлексивному управлению.

Экстремальные ситуации всегда связаны с неоднозначностью интерпретации условий и осознанием ограниченности действий привычными способами, субъект вынужден действовать на пределе своих психологических возможностей. Для субъекта, оказавшегося в чрезвычайных, опасных и экстремальных условиях ситуация является экстремальной (Магомед-Эминов, 2006; Шойгу, 2019), но при интерпретации этих условий как обычных, в которых субъект действует привычными способами, чрезвычайные или опасные условия не приводят к экстремальной ситуации. Нормальные условия могут сосуществовать с экстремальной ситуацией в силу искажений и противоречивости информации, неоднозначности ее интерпретации, осознания ограниченности привычных способов действий, с особенностями «ожиданий» и высокими требованиями к реакциям. Имеются и другие факторы возникновения трудностей, связанные с состоянием, мотивацией, личностными особенностями, внутренним конфликтом смысловой структуры действий, совершенными и обнаруженными ошибками, действиями других лиц (Шойгу, 2019). Типы экстремальных ситуаций могут быть выделены по источникам трудностей, к ним относятся, в частности, ситуации напряженные, проблемные, принятия решения, риска.

Напряженные ситуации характеризуются активацией дополнительных ресурсов выполнения и контроля действий. При высокой динамичности условий интерпретация осложняется необходимостью не только обнаружения, но и прогнозирования изменений. При высокой значимости и требованиях к характеристикам действий субъект осознает ограниченность своих ресурсов, но продолжает действовать на пределе возможностей. Показателями напряженности ситуаций являются характеристики функциональных систем (сенсорная, умственная, моторная напряженности), организации и контроля действий, функциональное состояние. В первую очередь напряженность проявляется в перцептивных процессах, распространяется на интеллектуальные процессы, процессы эмоциональной и сознательной регуляции, затрагивает и мотивационную сферу, определяется как специфическая психическая напряженность. Физические (статические и динамические) перегрузки, нервнопсихическое напряжение анализаторов, поддержание активности при монотонности труда проявляются в характеристиках функционального состояния и относятся к неспецифической напряженности. Напряженные ситуации, определяемые субъективной оценкой отношений, могут возникать у участников совместной деятельности. 
Ситуации неопределенности выделяются как класс по степени свободы действий, допускаемой условиями и наличными ресурсами. Как правило, неопределенность относится к будущему, однако характеризует и текущее состояние, если субъект не имеет возможности оценить условия с достаточной точностью и контролировать их. В детерминированных условиях, при наличии знаний о возможных изменениях и владении способами действий, при адекватной оценке условий и собственных возможностей, неопределенности не возникает, ситуация является обычной. При множественности или отсутствии вариантов интерпретации условий, оценки возможных состояний объекта, способов действий и результатов ситуация характеризуется неопределенностью.

Представления о неопределенности не отличаются однозначностью. Формальные определения (например, мера информации, состояние системы по отношению к «идеальным» детерминированным условиям или неоднозначность реализации событий) базируются на показателях достоверности, надежности и полноты данных. Источники неопределенности при этом связываются с условиями, претендуя на объективность описания информационной (в том числе прагматической и семантической) и временно́й неопределенности. Иногда определения фиксируют характеристику состояния субъекта «недостаток уверенности» в правильности оценок и понимания ситуации, что отражает лишь отношение к трудностям, не является необходимым сопровождением неопределенности. Психологическое содержание неопределенности ограничивается психической сферой, характеризует основания и возможности познавательных процессов, обеспечивающих интерпретацию условий, оценку полноты, достоверности, релевантности и значения информации, а также прогноза их изменений (Солнцева, 2019). Неопределенность - интегральная характеристика отношения (оценки) субъекта к среде, фиксирующая невозможность обобщенной оценки условий имеющимися ресурсами или оценки собственных ресурсов. Практически никогда нет всей необходимой информации для однозначного «определения» ситуации, но степень и содержание неопределенности могут быть разными. Неизвестными могут быть параметры объекта, способы действий и результат, какие состояния вообще он может принимать, каковы его связи с другими объектами, неизвестны и критерии их оценки. Субъект оказывается в ситуации высокой степени неопределенности, когда нет вариантов приемлемой интерпретации условий, отсутствуют и варианты действий. Он может знать, какие состояния объекта и способы их изменений возможны, но неизвестно, какое из возможных состояний характерно для данного момента, какой из известных способов является наилучшим. По характеру (степени) неопределенности все ситуации неопределенности можно разделить на два класса. Ситуации, в которых субъект не имеет вариантов интерпретации (и оценки), необходимо найти хотя бы один вариант приемлемого понимания объекта, - проблемные ситуации. Ситуации, в которых субъект располагает несколькими вариантами интерпретации, неизвестны лишь их преимущества и ограничения, - ситуации принятия решения. Отметим, что проблемная ситуация может на определенном этапе 
включать принятие решения, а в ситуации принятия решения могут потребоваться более глубокий анализ и решение возникшей проблемы.

Проблемная ситуация отличается осознанием ограниченности личностных ресурсов, невозможности достижения желаемого результата имеющимися способами. При наличии опыта и адекватной его оценке или при выполнении действий на уровне автоматизма проблемной ситуации не возникает. Ситуация возникает только при значимости результата и осознании конфликта между имеющимся опытом и требуемым результатом. При полном отсутствии знаний проблема не возникает, для оценки необходимы предварительные частичные знания и определенное незнание, соотношение которых инициирует познавательную активность. Проблема предстает как «знание о незнании» в результате осознания несоответствия желаемого и существующего. Субъект вынужден искать причины возникшего рассогласования, новые связи элементов ситуации, новые варианты «определения» ситуации, что предполагает новое целеполагание и активизацию процесса мышления. Осознание проблемы является начальным моментом мышления, а действия в проблемной ситуации приобретают признаки познавательной деятельности: выраженная познавательная мотивация, конкретизация познавательной цели и оценка ее значимости, личностная пристрастность, сознательный уровень регуляции. Проблемная ситуация является условием исследования мышления, а познавательная потребность приобретает характер отличительного признака проблемной ситуации. Характеристиками и показателями проблемной ситуации являются значимость проблемы по эмоциональному отношению и рациональной оценке, осознание ограниченности знаний и необходимости их восполнения. Адекватная оценка имеющихся ресурсов (возможностей) вызывает мобилизацию сил, а разрешение противоречия между знанием и незнанием обеспечивает формирование новых знаний и способов действий, неадекватная сопровождается снижением познавательной мотивации и вероятности успеха.

Проблемные ситуации могут быть напряженными в зависимости от отношения знания к незнанию: чем меньше рассогласование и ниже его оценка, больше совпадений с ситуациями прошлого опыта, тем менее напряженной является ситуация. Для познавательных видов деятельности проблемные ситуации приобретают характер обычных, успешность действий в проблемных ситуациях определяется интеллектуальными особенностями, в частности когнитивной сложностью.

Ситуации принятия решения относятся к ситуациям неопределенности при множественности вариантов и необходимости выбора. Выбор является необходимой, но недостаточной характеристикой ситуации, поскольку не всякий выбор связан с принятием решения. Не обсуждая подходов и аспектов проблем выбора, ограничимся пониманием выбора как формы активности, обеспечивающей сокращение множественности когнитивных и исполнительных схем в актуальных условиях. Выбор может приобретать характер операции с сознательным контролем результата, по терминологии В. Лефевра, по механизму быстрой рефлексии, или действия с развернутым анализом имеющихся 
когнитивных схем интерпретации и оценки условий, соотносимых с исполнительными схемами. Только сознательно регулируемое действие устранения неопределенности, обеспечивающее переход от множества возможностей (альтернатив) к определенности действия (решения), определяется как принятие решения (Солнцева, 1999).

Представление о необходимых и достаточных компонентах принятия решения (альтернативы, исходы, критерии, правила) разделяется практически всеми исследователями (Солнцева, 2019). Основной единицей активности является оценка условий, возможного будущего результата (исхода), способов и помех его достижения, реакции социального окружения, отдаленных последствий и др. Условием такой многокритериальной прогностической оценки является репрезентация всех компонентов ситуации в едином «пространстве непространственных явлений», в рефлексивном пространстве, фиксирующем значения элементов ситуации и формирующем смысл. Следствием отнесения принятия решения к рефлексивному пространству является особенность его процессного описания. Процесс не может быть описан как последовательность стадий, но могут быть представлены принципы его организации (Карпов, 2004). Принцип достаточной дифференциации предполагает, что процесс ограничивается необходимыми и достаточными критериями оценки. В обычных ситуациях процесс предстает в минимально развернутом виде перехода от интерпретации условий к решению, а в ситуациях затруднений процесс приобретает развернутую форму, включая, например, операции ранжирования критериев, оценки соответствия социальным нормам, позиции значимого другого. Принцип целевой детерминации означает, что процесс анализа вариантов развертывается от цели как образа результата к анализу ситуации, обеспечивая тем самым соподчиненность всех этапов решения, а соответствующие операции по степени значимости образуют структуру иерархического типа (принцип иерархичности). Рефлексивный анализ предполагает также многократные возвраты к отдельным операциям, изменение оценок и преобразование связей элементов ситуации по принципу итеративности.

Рефлексивная природа принятия решения проявляется в его выраженной личностной детерминации. При оценке альтернатив используется субъективная вероятность, которая не совпадает с объективной вероятностью в силу различий шкал измерения и оценки. Шкала объективной вероятности линейная с фиксированными крайними точками; субъективная оценочная носит нелинейный характер: объективной вероятности 0.5 соответствует субъективная вероятность 0.62 (Лефевр, 2003), а ее крайние точки являются скорее пределами, чем численными значениями, допускающими возможность явлений, которых объективно быть не может и «случайного» отклонения от известных законов. Обусловленные опытом оценки вероятности событий преувеличиваются, если субъект часто встречался со схожими ситуациями, он их легко припоминает и использует для идентификации. Вариативность оценок вероятности событий обусловлена также ошибками восприятия и мышления (в частности, трактовкой отношений альтернативности как транзитивных), переоценкой надежности прогнозов, особенностями мотивации, 
чувствительности к языку описания исходов и другими переменными (Канеман и др., 2005).

Предпочтения в выборе альтернатив обусловлены значимостью, ожидаемой полезностью и ценой исходов: чем выше значимость, тем выше «цена», которую человек готов заплатить, выше субъективная оценка вероятности положительного исхода и выше оценка приемлемости отрицательного исхода. Если «цена» затрат минимальна, вероятность положительного исхода оценивается на порядок выше. Полезность и цена - оценки собственных устремлений и имеющихся ресурсов - зависят от системы ценностей и самооценки: высокая самооценка расширяет количество приемлемых альтернатив и возможных вариантов действий, низкая детерминирует сокращение рассматриваемых вариантов за счет исключения «недостижимых». Отмечено, что стремление к успеху проявляется в выборе вариантов средних значений оценки альтернатив, при избегании неудач предпочтение отдается альтернативам с крайними значениями (Макклелланд, 2007).

Проблемные ситуации и ситуации принятия решения имеют существенные различия. В проблемных ситуациях опыт ограничен, а его концептуальная избыточность затрудняет поиск приемлемого варианта интерпретации и оценки условий, в принятии решений опыт - условие выбора, а его полнота сказывается на выборе лучшего варианта. Решение проблем - когнитивная задача - обеспечивается мыслительными процессами; принятие решения рефлексивная оценочная задача даже в случаях, когда альтернативы не заданы и не очевидны. Процесс решения проблем может быть описан последовательностью этапов, действиями и операциями; при принятии решений оценки вариантов не имеют временно́й отнесенности. Проблемная ситуация характеризуется возникновением познавательной потребности, приобретает признаки деятельности; принятие решения не имеет специфической мотивации, является особым действием с функцией формирования актуальной структуры (актуалгенеза или системогенеза) деятельности (Карпов, 2003), а также функцией регуляции на стадии реализации. Результатом решения проблемы является новое знание вне зависимости от языка его описания; в принятии решения - вариант организации действия по целевой функции, зависящий от языка описания альтернатив (достижений или потерь). Различие ситуаций проявляется в конфигурации личностных детерминант: решающими для решения проблем являются интеллектуальные способности, для принятия решения - уровень рефлексии.

Риск и ситуации риска. В широком контексте и обыденном сознании риск связывается с возможностью отрицательного воздействия, результата деятельности или потерь, в явном виде отождествляется, ограничивается и измеряется вероятностью неблагоприятных последствий. Изменения условий, например наводнения, возможные события страхового случая, финансового кризиса, заболевания или возможность неблагоприятного результата действия, оцениваются вероятностью и определяются как риск (Ильин, 2012), что в практике управления организациями закреплено задачей «оценки и управления рисками». В информационном подходе риск определяется как 
атрибутивная характеристика и мера неопределенности: с повышением неопределенности возрастает и риск. Отличие риска от неопределенности сводится к объему доступной информации: отсутствие информации характеризует неопределенность, ее наличие соответствует понятию риска как «измеримой неопределенности», а процесс восполнения информации определяется как переход неопределенности в риск. Семантическое несовпадение риска и вероятности отрицательного исхода отразилось в их трактовке даже в рамках формальных представлений. В оценочном подходе за счет введения субъективной переменной - отношения к результату (исходу) - неопределенность характеризует нейтральное отношение, риск ограничивается позитивным отношением к отрицательному исходу. Для психологического анализа именно субъективные переменные оценки и отношения к исходам, а не их вероятность обуславливают выбор и риск.

Риск - не вероятность отрицательного результата, а характеристика действия, приведшего к выбору варианта с высокой вероятностью отрицательного исхода (Солнцева, 1999), проявляющаяся в «управлении» вероятностью исходов. В условиях опасности, например, выбранный вариант действия характеризуется уменьшением или увеличением вероятности неблагоприятного исхода при удалении или приближении к источнику опасности. Вероятность отрицательного исхода является мерой риска, хотя субъект может вообще не располагать данными, позволяющими оценить вероятность, использовать эмоциональное или социально-нормативное отношения в качестве критериев оценки (Солнцева, 2019).

Ситуация риска - частный случай ситуации принятия решения, характеризующийся высокой вероятностью отрицательного исхода при значимости и ориентации на достижение положительного результата. При субъективной вероятности $\mathrm{P} \geqslant 0.5$ положительного исхода (соответствующей объективной $\mathrm{P} \geqslant 0.38$ ) (Лефевр, 2003) нет необходимости оценивать неблагоприятный исход, предпочтения отдаются варианту с большей вероятностью. Формальные основания рационального выбора предполагают отказ от альтернатив с низкой вероятностью положительного результата, однако при вероятности $\mathrm{P} \leqslant 0.5$ субъект остается в ситуации неопределенности, вынужден оперировать показателями положительных и отрицательных исходов, пытаясь преодолеть противоречие высокой значимости и низкой вероятности положительного исхода. Задача усложняется, приобретая характер многокритериального выбора, ее решение требует расширения арсенала критериев и интеграции оценок исходов альтернативы.

Выбор обусловлен не столько осознанием возможности неблагоприятного исхода, сколько оценкой отношения значимости положительного и отрицательного исходов альтернативы, личностного отношения необходимости, желательности, значимости, полезности ожидаемого положительного результата к требуемым ресурсам, потерям, затратам для его достижения. Субъект оценивает, по сути, отношение объективных характеристик условий (в субъективной интерпретации) и собственного состояния - ресурсов, возможностей, которое приобретает характер интегрального критерия выбора - принять 
альтернативу и действовать или отказаться от нее, искать другие способы достижения цели или оправдать свой отказ. Фактором выбора, как и любой ситуации, является адекватность оценок условий и ресурсов в пределах актуальной мотивации и опыта действий, сложившихся правил соотнесения и интеграции оценок, упрощения ситуации и преодоления неопределенности.

Таким образом, ситуации риска в классе ситуаций принятия решения ограничены представлением о предпочтении выбора альтернативы, отличающейся высокой значимостью и низкой вероятностью положительного результата (исхода), а понятие риска приобретает смысл характеристики действия (выбора) в ситуации принятия решения: чем выше вероятность отрицательного исхода, тем больше риск.

Диапазон возможных ситуаций риска в деятельности можно представить тремя зонами оценок отношений полезности к потерям. Область ожиданий высокой полезности при достаточности ресурсов и оценке потерь как незначительных и восполнимых - зона приемлемого риска. При оценке относительного равенства высокой значимости и больших потерь выбор предполагает «согласие» субъекта с возможными потерями и усилиями (ценой), риск считается допустимым. При высокой вероятности неблагоприятного исхода и высокой цене альтернатива может не исключаться как неприемлемая в силу высокой значимости и «шанса» положительного исхода, риск признается обоснованным. Все остальные случаи могут рассматриваться как необоснованный риск, связанный с особенностями мотивации, неадекватной оценкой возможностей, ограниченностью опыта.

Ситуации риска преимущественно характеризуются напряженностью, требуют актуализации дополнительных ресурсов для достижения желаемого результата. Личностная обусловленность риска как характеристика действий субъекта проявляется в феноменах и парадоксах действий в ситуациях риска: придание большей значимости оценкам и суждениям, чем какому-либо другому виду расчета, бо́льшая чувствительности к потерям, чем к выигрышам, снижение оценок потерь при их возрастании, зависимость решений от формулировки условий. Характеристики приемлемости, допустимости и обоснованности рисков связываются с представлениями о склонности, готовности к риску и принятию риска, диагностируются на основе отчетов испытуемых о типичном или предпочитаемом для них поведении в предлагаемых ситуациях. Эти особенности являются лишь поведенческими проявлениями предпочтений, обусловленными структурой базовых личностных качеств, исследование которой было и остается одной из центральных проблем психологии.

\section{Заключение}

Ограничение понятия ситуации рамками субъективной реальности снимает противопоставление и необходимость оценивать преобладание средового или личностного факторов, выделять объективную и субъективную ситуации, тем более объяснять «взаимодействие личности и ситуации». Направленность ситуационного подхода - исследование содержания рефлексивного 
образа как результата взаимодействия субъекта с условиями среды, ситуация становится основной единицей анализа действий, позволяющей выявлять трудности и причины ошибок, устранение которых и поддержка действий обеспечивают готовность к деятельности в различных условиях.

Рассмотренные ситуации не ограничивают их спектр, перспективным представляется описание особенностей кризисных и критических ситуаций, ситуаций совместной деятельности, конфликта, воздействия и рефлексивного управления. Систематизация как необходимое условие научного исследования предполагает выявление психологических механизмов и закономерностей реализации деятельности в разных условиях и ситуациях. Значимость подхода видится в том, что именно анализ ситуации является основой прогноза поведения субъекта, разработки мер снижения негативных ситуативных проявлений, повышения психологической готовности к действиям. Все экстремальные ситуации характеризуются ограниченностью ресурсов - характеристик функциональных систем и опыта. Ограниченность первых преодолевается за счет совершенствования технических средств взаимодействия со средой, предоставления достоверной, релевантной, необходимой и достаточной информации при оптимизации временно́го режима ее поступления. Опыт остается основным фактором адекватной оценки условий, собственных возможностей, интеграции оценок, определения ситуации как обычной и использования сложившихся привычных способов действия. Особого внимания требуют проблемы содержания и структуры опыта, диагностики индивидуальных причин затруднений для успешной деятельности в различных ситуациях.

\section{Литература}

Анцупов, А. Я., Шипилов, А. И. (2010). Словарь конфликтолога (3-е изд., испр. и доп.). М.: Эксмо. Воронин, В. Н., Князев, В. Н. (1989). К определению психологического понятия ситуации. В кн. Актуальные вопросы организачионно-психологческого обеспечения работь с кадрами (с. 121-126). М.: Российское педагогическое агентство.

Василюк, Ф. Е. (2003). Методологический анализ в психологии. М.: МГППУ; Смысл.

Гришина, Н. В. (2008). Психология конфликта (2-е изд.). СПб.: Питер.

Ильин, Е. П. (2012). Психология риска. СПб.: Питер.

Канеман, Д., Словик, П., Тверски, А. (2005). Принятие решений в неопределенности: Правила и предубеждения. Харьков: Гуманитарный центр.

Карпов, А. В. (2003). Психология принятия решения. Ярославль: Институт психологии РАН; Ярославский государственный университет.

Карпов, А. В. (2004). Психология рефлексивных механизмов деятельности. М.: Изд-во «Институт психологии РАН».

Лебедев, В. И. (1989). Личность в экстремальных условиях. М.: Политиздат.

Лефевр, В. А. (2003). Рефлексия. М.: Когито-центр.

Ломов, Б. Ф. (1999). Методологические и теоретические проблемы психологии. М.: Наука.

Лысаков, Н. Д., Гандер, Д. В., Лысакова, Е. Н. (2013). Психология труда в экстремальных условиях: монография. М.: Изд-во СГУ. 
Магнуссон, Д. (1983). Ситуационный анализ: Эмпирические исследования соотношений выходов и ситуаций. Психологический журнал, 2, 29-54.

Магомед-Эминов, М. Ш. (2006). Экстремальная психология. М.: ПАРФ.

Макклелланд, Д. (2007). Мотивация человека. СПб.: Питер.

Осухова, Н. Г. (2012). Психологическая помощь в трудных и экстремальных ситуациях (5-е изд., перераб. и доп.). М.: Издательский центр «Академия».

Попова, Р. Р. (2011). Проблема определения понятия «событие» в психологии. Вестник Татарского государственного гуманитарно-педагогического университета, 3(25), 287-293.

Рягузова, Е. В. (2006). Ситуация: горизонты психологической интерпретации. Известия Саратовского университета. Новая серия. Серия: Психология. Философия. Педагогика, 6(1), 81-87.

Солнцева, Г. Н. (1999). Риск как характеристика действий субъекта. М.: Изд-во Московского университета.

Солнцева, Г. Н. (2019). Принятие решения в профессиональной деятельности. В кн. Е. А. Климов, О. Г. Носкова, Г. Н. Солнцева (ред.), Психология труда, инженерная психология и эргономика: Ч. 2. Учебник для академического бакалавриата (с. 55-74). М.: Юрайт.

Трифонова, С. А. (2004). Психология социальных ситуаций. Ярославль: Ярославский государственный университет.

Филиппов, А. В., Ковалев, С. В. (1986). Ситуация как элемент психологического тезауруса. Психологический журнал, 1, 14-21.

Швырев, В. С. (1985). Рефлексия и понимание в современном анализе науки. Вопросы философии, 5, 44-56.

Шойгу, Ю. С. (ред.). Психология экстремальных ситуаций. М.: Смысл.

Ссылки на зарубежные источники см. в разделе References после англоязычного блока.

Солнцева Галина Николаевна - доцент, факультет психологии, Московский государственный университет имени М.В. Ломоносова, кандидат психологических наук.

Сфера научных интересов: структура и регуляция деятельности, профессиональная деятельность.

Контакты: galinasolntseva@mail.ru 


\title{
Situational Approach: Types of Situations and Psychological Characteristics
}

\author{
G.N. Solntseva ${ }^{a}$ \\ ${ }^{a}$ Lomonosov Moscow State University, 1 Leninskie Gory, Moscow, 119991, Russian Federation
}

\begin{abstract}
In a wide range of psychological approaches, a situational approach, which has received theoretical justification in domestic studies, is promising. The central concept of "situation" is defined as an indirect element between the conditions of the environment and the individual personality characteristics of the subject in the context of problems of double determination of activity. Definitions of the situation are mostly limited to the idea of a subjective image of external conditions, the adequacy of which is interpreted as a manifestation of the environmental determination of activity, while its variability is understood as the manifestation of personal determination of activity. The partiality of the image of external conditions is one of the manifestations of personal determination of the cognitive component, which does not rule out the problem of determination of activity. The purpose of the work is to justify limitation of the concept of "situation" to the subjective sphere and to interpret it as the result of integration of the determinants. The notions of the environment are clarified; the need for assessments of the interpreted image of conditions, of subject's own resources and their balance, as well as of the "room" for appraisals and integration of the determinants, are rationalized. The situation is defined as a reflexive appraisal "model" of the relationship between the interpreted image of objective conditions and the internal state, within the limits of actual motivation, actual experience and personal preferences. The functions of the situation are the organization of activity (actual-genesis) and its regulation at the stage of execution, ensuring its stability and expediency. Possible systematization of situations based on the typology of conditions and options for their subjective assessment, the subject's possibilities and their assessment, is presented. Particular attention is paid to the situations of uncertainty, decision-making and risk; their features, psychological mechanisms and personal determinants are described. The situational approach enhances the possibilities of explaining the variability of activity in identical conditions, its sustainability in changing conditions, as well as explaining the causes of errors caused by the uniqueness of the assessments in a reflexive model of reality.
\end{abstract}

Keywords: environment, conditions, situation, emergency, complexity, extreme, danger, tension, uncertainty, problem, decision-making, risk, reflection.

\section{References}

Antsupov, A. Ya., \& Shipilov, A. I. (2010). Slovar' konfliktologa [Conflictologist's dictionary] (3rd ed.). Moscow: Eksmo.

Filippov, A. V., \& Kovalev, S. V. (1986). Situatsiya kak element psikhologicheskogo tezaurusa [Situation as an element of psychological thesaurus]. Psikhologicheskii Zhurnal, 1, 14-21. 
Grishina, N. V. (2008). Psikhologiya konflikta [The psychology of conflict] (2nd ed.). Saint Petersburg: Piter. Il'in, E. P. (2012). Psikhologiya riska [The psychology of risk]. Saint Petersburg: Piter.

Kahneman, D., Slovik, P., \& Tversky, A. (2005). Prinyatie reshenii v neopredelennosti: Pravila i predubezhdeniya [Judgment Under Uncertainty: Heuristics and biases]. Kharkiv, Ukraine: Gumanitarnyi tsentr. (Original work published 1982)

Karpov, A. V. (2003). Psikhologiya prinyatiya resheniya [The psychology of decision-making]. Yaroslavl: Institute of Psychology of the RAS; Yaroslavl State University.

Karpov, A. V. (2004). Psikhologiya refleksiznykh mekhanizmov deyatel'nosti [The psychology of reflexive mechanisms of activity]. Moscow: Institute of Psychology of the RAS.

Lebedev, V. I. (1989). Lichnost'v ekstremal'nykh usloviyakh [Personality in extreme conditions]. Moscow: Politizdat.

Lefebvre, V. A. (2003). Refleksiya [Reflexion]. Moscow: Kogito-tsentr.

Lomov, B. F. (1999). Metodologicheskie i teoreticheskie problemy psikhologii [Methodological and theoretical issues of psychology]. Moscow: Nauka.

Lysakov, N. D., Gander, D. V., \& Lysakova, E. N. (2013). Psikhologiya truda v ekstremal'nykh usloziyakh [The psychology of labor in extreme conditions]. Moscow: SGU.

MacClelland, D. (2007). Motivatsiya cheloveka [Human motivation]. Saint Petersburg: Piter. (Original work published 1987)

Magnusson, D. (1983). Situatsionnyi analiz: Empiricheskie issledovaniya sootnoshenii vykhodov i situatsii [Situational analysis: Empirical studies of the balance between the outcomes and the situations]. Psikhologicheskii Zhurnal, 2, 29-54.

Magomed-Eminov, M. Sh. (2006). Ekstremal'naya psikhologiya [Extreme psychology]. Moscow: PARF.

Osukhova, N. G. (2012). Psikhologicheskaya pomoshch'v trudnykh i ekstremal'nykh situatsiyakh [Psychological help in difficult and extreme situations] (5th ed.). Moscow: Izdatel'skii tsentr "Akademiya".

Popova, R. R. (2011). The problem of definition of the concept "event" in psychology. Vestnik Tatarskogo Gosudarstvennogo Gumanitarno-Pedagogicheskogo Universiteta, 3(25), 287-293. (in Russian)

Ryaguzova, E. V. (2006). Situatsiya: gorizonty psikhologicheskoi interpretatsii [Situation: horizons of psychological interpretation]. Izvestiya Saratovskogo universiteta. Novaya seriya. Seriya: Psikhologiya. Filosofiya. Pedagogika, 6(1), 81-87.

Shoigu, Yu. S. (Ed.). Psikhologiya ekstremal'nykh situatsii [The psychology of extreme situations]. Moscow: Smysl.

Shvyrev, V. S. (1985). Refleksiya i ponimanie v sovremennom analize nauki [Reflexion and understanding in contemporary analysis of science]. Voprosy Filosofii, 5, 44-56.

Solntseva, G. N. (1999). Risk kak kharakteristika deistvii sub"ekta [Risk as a characteristic of subject's acts]. Moscow: Moscow University Press.

Solntseva, G. N. (2019). Prinyatie resheniya v professional'noi deyatel'nosti [Decision-making in professional activity]. In E. A. Klimov, O. G. Noskova, \& G. N. Solntseva (Eds.), Psikhologiya truda, inzhenernaya psikhologiya i ergonomika [Labor psychology, engineering psychology and ergonomics] (Pt. 2, pp. 55-74). Moscow: Yurait.

Trifonova, S. A. (2004). Psikhologiya sotsial'nykh situatsii [The psychology of social situations]. Yaroslavl: Yaroslavl State University.

Vasilyuk, F. E. (2003). Metodologicheskii analiz v psikhologii [Methodological analysis in psychology]. Moscow: MGPPU; Smysl. 
Voronin, V. N., \& Knyazev, V. N. (1989). K opredeleniyu psikhologicheskogo ponyatiya situatsii [On the definition of the psychological notion of situation]. In Aktual'nye voprosy organizatsionnopsikhologicheskogo obespecheniya raboty s kadrami [Current issues of staff development in organizational psychology] (pp. 121-126). Moscow: Rossiiskoe pedagogicheskoe agentstvo.

Galina N. Solntseva - Associate Professor, Department of Psychology, Lomonosov Moscow State University, $\mathrm{PhD}$ in Psychology.

Research Area: structure and regulation of activities, professional activity.

E-mail: galinasolntseva@mail.ru 